TRANSACTIONS OF THE

AMERICAN MATHEMATICAL SOCIETY

Volume 360, Number 12, December 2008, Pages 6695-6709

S 0002-9947(08)04526-1

Article electronically published on July 24, 2008

\title{
A COMBINATORIAL METHOD FOR CALCULATING THE MOMENTS OF LÉVY AREA
}

\author{
DANIEL LEVIN AND MARK WILDON
}

\begin{abstract}
We present a new way to compute the moments of the Lévy area of a two-dimensional Brownian motion. Our approach uses iterated integrals and combinatorial arguments involving the shuffle product.
\end{abstract}

\section{INTRODUCTION}

In this paper we present a new approach to the problem of finding the moments of the signed area swept out by a two-dimensional Brownian motion. This is a classical problem of great importance, originally solved by Lévy (see [10]).

We begin by explaining how these moments may be defined. Given a piecewise smooth path $\gamma_{t}:[0, T] \rightarrow \mathbf{R}^{2}$ we may complete it to a loop $\bar{\gamma}$ by closing it with the chord from $\gamma_{T}$ to $\gamma_{0}$. We may then define its signed area to be

$$
\iint_{\mathbf{R}^{2}} n(\bar{\gamma}, x) d x
$$

where $n(\bar{\gamma}, x)$ is the winding number of $\bar{\gamma}$ about the point $x \in \mathbf{R}^{2}$. (See Figure 1.)

Now suppose that $B_{t}:[0, T] \rightarrow \mathbf{R}^{2}$ is a two-dimensional Brownian motion. If we complete $B$ to a loop $\bar{B}$ and attempt to define the signed area of $B_{t}$ as before, then we immediately encounter the problem that, almost surely, $n\left(\bar{B}_{t}, \cdot\right)$ is not integrable on $\mathbf{R}^{2}$ (see [9, Theorem 55.I]). One solution is to replace $B_{t}$ with a sequence of piecewise linear dyadic approximations. In [8, Theorem 10] and [9, Chapter 55], Lévy proved that, almost surely, the winding number integral is defined for each approximation, and that the sequence of areas converges. This gives one possible definition of the Lévy area of the process $B_{t}$.

We may also define the signed area of a smooth path $\gamma:[0, T] \rightarrow \mathbf{R}^{2}$ with $\gamma_{t}=$ $\left(x_{t}, y_{t}\right)$ by

$$
\frac{1}{2} \int_{0}^{T}\left(\left(x_{s}-x_{0}\right) y_{s}^{\prime}-\left(y_{s}-x_{0}\right) x_{s}^{\prime}\right) d s .
$$

This observation motivates the following alternative definition of Lévy area.

Received by the editors February 1, 2007 and, in revised form, April 16, 2007.

2000 Mathematics Subject Classification. Primary 60J65; Secondary 05A15.

Key words and phrases. Lévy area, shuffle product, signature of a path.

The first author was supported by the EPSRC Fellowship "Partial differential equations - A rough path approach" GR/S18526/01.

The second author was supported by EPSRC Grant EP/D054664/1. 


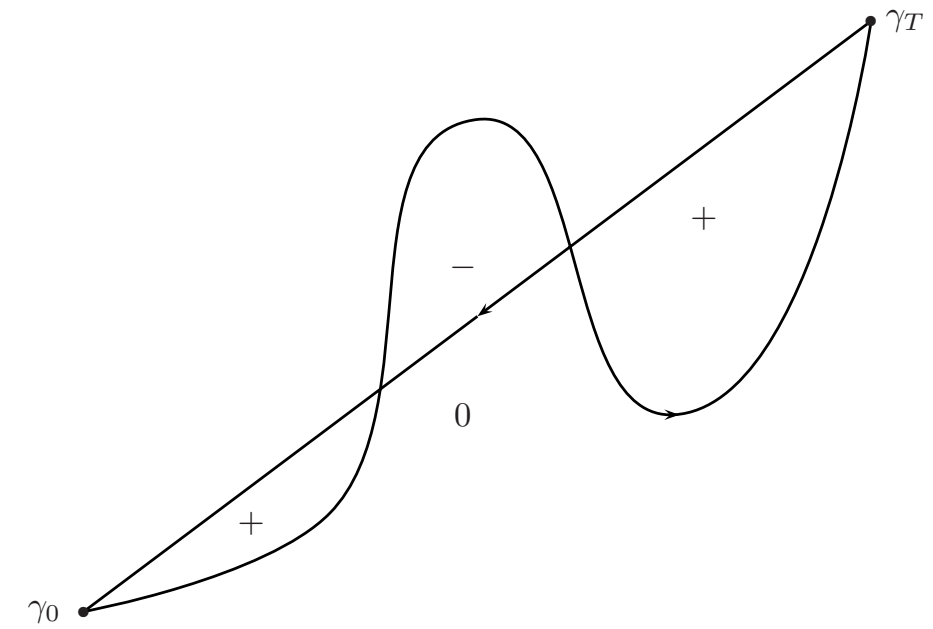

Figure 1. Contributions to the signed area of $\gamma:[0, T] \rightarrow \mathbf{R}^{2}$.

Definition 1.1. Let $B_{t}=\left(X_{t}, Y_{t}\right)$ for $t \in[0, T]$ be a two-dimensional Brownian motion starting at 0 . The Lévy area of $\left(B_{t}\right)_{0 \leq t \leq T}$ is given by the stochastic integral

$$
\mathcal{A}_{T}=\frac{1}{2} \int_{0}^{T}\left(X_{s} d Y_{s}-Y_{s} d X_{s}\right) .
$$

Remark 1.2. In the sequel, we use $\mathcal{A}$ as a shorter notation for $\mathcal{A}_{1}$.

Lévy showed in [10] that almost surely the definitions of signed area by dyadic approximation and by stochastic integration agree. In his paper he also gave two different ways to find the characteristic function, and hence the moments, of $\mathcal{A}_{T}$ when $T=2 \pi$.

Theorem 1.3 (Lévy). If $T=2 \pi$, then

$$
\mathbb{E}\left(\exp \left(i z \mathcal{A}_{2 \pi}\right)\right)=(\cosh \pi z)^{-1} .
$$

Lévy's first proof uses the definition of $\mathcal{A}_{T}$ by dyadic approximation. His second starts from Definition 1.1. but depends on earlier work by Kac, Siegert, Cameron and Martin (see references cited in [10, §1.6]).

In this paper we present a direct and largely self-contained proof of Levy's Theorem, using Definition 1.1 to define Lévy area. Our approach, which we outline in $\S 2$ below, is based on the fact that moments of Lévy area can be expressed as iterated integrals and hence calculated explicitly by exploiting the combinatorics of shuffle products.

It seems likely that our methods can be applied more broadly. We mention here that by using the multiplication

$$
(x, y, a)\left(x^{\prime}, y^{\prime}, a^{\prime}\right)=\left(x+x^{\prime}, y+y^{\prime}, a+a^{\prime}+\frac{1}{2}\left(x y^{\prime}-y x^{\prime}\right)\right),
$$

we may identify points $(x, y, a) \in \mathbf{R}^{3}$ with elements of the Heisenberg group. It is known (see [12, Section 3.2.2]) that the process $\left(X_{t}, Y_{t}, \mathcal{A}_{t}\right)$ is a Brownian motion on this group. Computing aspects of the joint distribution of $\left(X_{t}, Y_{t}, \mathcal{A}_{t}\right)$ is a hard problem, involving Harish-Chandra formulae (see [2]); it is in effect aimed at understanding the heat kernel on the Heisenberg group (see [4]). It seems likely 
that our approach may shed some light on these questions. Moreover, it should be possible to use our methods to study the signed areas obtained when we replace Brownian motion by measures related to higher order PDEs.

\section{Outline}

The outline of our proof is as follows. In $\S 3$ we use a simple scaling argument to show that Theorem 1.3 is implied by the following assertion about the moments of $\mathcal{A}$.

Theorem 2.1. $\mathbb{E} \mathcal{A}^{n}=2^{-n} E_{n}$.

Here $E_{n}$ is the $n$th Euler number, as defined by the generating function

$$
\sum_{n=0}^{\infty} E_{n} \frac{z^{n}}{n !}=(\cos z)^{-1}
$$

The first few non-zero Euler numbers are $E_{0}=1, E_{2}=1, E_{4}=5, E_{6}=61$. Of course all odd-numbered Euler numbers are zero. (Correspondingly, one can easily see that $\mathbb{E} \mathcal{A}^{n}=0$ if $n$ is odd.) We then show that the moments of $\mathcal{A}$ can be expressed using iterated integrals.

In $\S 4$ we introduce the shuffle product on the tensor algebra of a vector space and use it to give an expression for $\mathbb{E} \mathcal{A}^{n}$ as a certain coefficient in the expansion of a shuffle product. In $\S 5$ we use a combinatorial argument to determine this coefficient, thereby proving Theorem 2.1 and hence Lévy's Theorem.

\section{The MOMEnts of LÉvy's AREA For Brownian MOtion}

We first show that Lévy's theorem (Theorem 1.3) follows from Theorem 2.1 If we scale the Brownian path $B_{t}=\left(X_{t}, Y_{t}\right)$ defined for $0 \leq t \leq 1$ by setting $\tilde{B}_{s}=\sqrt{T} B_{s / T}$, then we obtain a new Brownian path defined for $0 \leq t \leq T$. As before, $\mathcal{A}$ is the Lévy area of $B_{t}$ at time 1 and $\mathcal{A}_{T}$ is the Lévy area of $\tilde{B}_{s}$ at time $T$. It follows easily from Definition 1.1 that $\mathcal{A}_{T}=T \mathcal{A}_{1}$. Hence, assuming that Theorem 2.1 holds, the moments of Lévy area at time $T$ are given by

$$
\mathbb{E} \mathcal{A}_{T}^{n}=2^{-n} T^{n} E_{n}
$$

In particular, by setting $T=2 \pi$ we find that the characteristic function of Lévy area at time $2 \pi$ is

$$
\mathbb{E}\left(\exp \left(i z \mathcal{A}_{2 \pi}\right)\right)=\sum_{n=0}^{\infty} \pi^{n} E_{n} \frac{(i z)^{n}}{n !}=(\cos \pi i z)^{-1}=(\cosh \pi z)^{-1},
$$

where we have absolute convergence of the series for $|z|<1 / 2$.

Therefore, to prove Theorem 1.3, we may concentrate on finding the moments of $\mathcal{A}$. For this we shall need the following algebraic definition.

Definition 3.1. Let $V$ be a real vector space. Let

$$
T((V))=\prod_{k=0}^{\infty} V^{\otimes k},
$$

where by convention $V^{\otimes 0}=\mathbf{R}$. Clearly $T((V))$ is a real vector space. It is easy to check that $T((V))$ becomes an algebra with unit if we define the product of 


$$
\begin{aligned}
\mathbf{a}=\left(a_{0}, a_{1}, a_{2}, \ldots\right), \mathbf{b}=\left(b_{0}, b_{1}, b_{2}, \ldots\right) \in T((V)) \text { by } \\
\quad \mathbf{a} \otimes \mathbf{b}=\left(\ldots, \sum_{j=0}^{k} a_{j} \otimes b_{k-j}, \ldots\right) .
\end{aligned}
$$

We shall usually write the elements of $T((V))$ as formal infinite sums. Thus if $a_{k} \in V^{\otimes k}$, then the infinite sequence $\left(a_{0}, a_{1}, a_{2}, \ldots\right) \in T((V))$ will be denoted by $a_{0}+a_{1}+a_{2}+\cdots$. The reader may easily check that this convention is consistent with the vector space structure on $T((V))$. We use this convention to define the exponential of a tensor $a \in V^{\otimes k}$ as follows:

$$
\exp (a)=\sum_{n=0}^{\infty} \frac{a^{\otimes n}}{n !}
$$

We are now ready to define the signature of a Brownian motion. As motivation, we first recall this definition for a path. If $\gamma:[0, T] \rightarrow \mathbf{R}^{2}$ is a path of finite length, then its signature (see [6]) is the formal infinite sum

$$
\mathbf{X}_{s, t}(\gamma)=1+\sum_{k=1}^{\infty} \int_{s<t_{1}<\cdots<t_{k}<t} d \gamma_{t_{1}} \otimes \cdots \otimes d \gamma_{t_{k}} \in T\left(\left(\mathbf{R}^{2}\right)\right)
$$

defined for $0 \leq s<t \leq T$.

Example 3.2. If $e_{0}, e \in \mathbf{R}^{2}$ and $\gamma_{t}=e_{0}+t e$, then

$$
\begin{aligned}
\mathbf{X}_{s, t}(\gamma) & =1+\sum_{k=1}^{\infty} \int_{s<t_{1}<\cdots<t_{k}<t} d \gamma_{t_{1}} \otimes \cdots \otimes d \gamma_{t_{k}} \\
& =1+\sum_{k=1}^{\infty} \int_{s<t_{1}<\cdots<t_{k}<t} e d t_{1} \otimes \cdots \otimes e d t_{k} \\
& =1+\sum_{k=1}^{\infty} e^{\otimes k} \int_{s<t_{1}<\cdots<t_{k}<t} d t_{1} \cdots d t_{k} \\
& =1+\sum_{k=1}^{\infty} \frac{e^{\otimes k}}{k !}(t-s)^{k} \\
& =\exp ((t-s) e) .
\end{aligned}
$$

Here we used the easily verified fact that

$$
\int_{s<t_{1}<\cdots<t_{k}<t} d t_{1} \ldots d t_{k}=\frac{(t-s)^{k}}{k !} .
$$

One of the most important properties of the signature is that it is multiplicative (for the proof of the following theorem, see [1]).

Theorem 3.3. Let $\gamma_{t}$ be a path of finite length defined for $0 \leq t \leq T$. If $0 \leq r<$ $s<t \leq T$, then

$$
\mathbf{X}_{r, t}(\gamma)=\mathbf{X}_{r, s}(\gamma) \otimes \mathbf{X}_{s, t}(\gamma)
$$

The most direct way to extend the definition of signature from paths of finite length to Brownian paths is to replace the iterated integrals in the definition already given with iterated stochastic integrals. See [5] for the details of this approach. This leads to the definition below. 
Definition 3.4. Let $B:[0, T] \rightarrow \mathbf{R}^{2}$ be a two-dimensional Brownian motion. For $0 \leq s<t \leq T$ we define the signature of $B$ to be the formal infinite sum of iterated stochastic integrals

$$
\mathbf{X}_{s, t}(B)=1+\sum_{k=1}^{\infty} \int_{s<t_{1}<\ldots<t_{k}<t} d B_{t_{1}} \otimes \cdots \otimes d B_{t_{k}} .
$$

Remark 3.5. The definition of Lévy area and the signature of a Brownian motion may be interpreted using either the Stratonovich or Itô stochastic integral. However, the next theorem holds only for the Stratonovich integral, so we shall work with this integral from now on.

The following theorem is proved in Fawcett [3] and in Lyons-Victoir [11.

Theorem 3.6. If $B$ is a Brownian motion in $\mathbf{R}^{2}$, then

$$
\mathbb{E}\left(\mathbf{X}_{0,1}(B)\right)=\exp \left(\frac{1}{2}\left(e_{1} \otimes e_{1}+e_{2} \otimes e_{2}\right)\right),
$$

where $e_{1}$ and $e_{2}$ are any two orthogonal vectors in $\mathbf{R}^{2}$.

In particular, (11) implies that

$$
\mathbb{E}\left(X_{0,1}^{2 n}(B)\right)=\frac{1}{2^{n} n !}\left(e_{1} \otimes e_{1}+e_{2} \otimes e_{2}\right)^{\otimes n},
$$

where $X_{0,1}^{2 n}$ is the component of $\mathbf{X}_{0,1}$ lying in $\left(\mathbf{R}^{2}\right)^{\otimes 2 n}$.

\section{Shuffle Products And other COMbinatorial objects}

We now introduce an important combinatorial object, which will be used in the sequel.

Definition 4.1. We define the set $S_{m, n}$ of $(m, n)$ shuffles to be the subset of permutations in the symmetric group $S_{m+n}$ defined by

$$
S_{m, n}=\left\{\sigma \in S_{m+n}: \sigma(1)<\cdots<\sigma(m), \sigma(m+1)<\cdots<\sigma(m+n)\right\} .
$$

Remark 4.2. The term "shuffle" is used because such permutations arise when one riffle shuffles a deck of $m+n$ cards cut into one pile of $m$ cards and a second pile of $n$ cards.

Let $V=\mathbf{R}^{2}$ with the orthogonal basis $e_{1}$ and $e_{2}$. Let $V^{*}$ be the space dual to $V$ and let $e^{1}$ and $e^{2}$ be its dual basis. Let $n \in \mathbf{N}$. The elements $e_{i_{1}} \otimes \cdots \otimes e_{i_{n}}$, where each $i_{k} \in\{1,2\}$, for $k=1, \ldots, n$, form a basis of $V^{\otimes n}$. The corresponding dual basis of $\left(V^{*}\right)^{\otimes n}$ is given by the elements $e^{i_{1}} \otimes \cdots \otimes e^{i_{n}}$.

There is a natural duality $\langle\rangle:, V^{\otimes n} \times\left(V^{\star}\right)^{\otimes n} \rightarrow \mathbf{R}$ defined by

$$
\left\langle e^{i_{1}} \otimes \cdots \otimes e^{i_{n}}, e_{j_{1}} \otimes \cdots \otimes e_{j_{n}}\right\rangle=\delta_{i_{1} j_{1}} \ldots \delta_{i_{n} j_{n}} .
$$

Definition 4.3. Set $\left(k_{1}, \ldots, k_{m+n}\right)=\left(i_{1}, \ldots, i_{m}, j_{1}, \ldots, j_{n}\right)$. The shuffle product of two tensors $e^{I}=e^{i_{1}} \otimes \cdots \otimes e^{i_{m}}$ and $e^{J}=e^{j_{1}} \otimes \cdots \otimes e^{j_{n}}$ is the tensor $e^{I} ш e^{J}$ defined by

$$
e^{I} ш e^{J}=\sum_{\sigma \in S_{m, n}} e^{k_{\sigma}-1(1)} \otimes \cdots \otimes e^{k_{\sigma}-1(m+n)} .
$$

Let $T\left(V^{\star}\right)=\bigoplus_{k=0}^{\infty}\left(V^{\star}\right)^{\otimes k}$ be the ordinary tensor algebra on $V^{\star}$. The shuffle product $\boldsymbol{\omega}$ extends to a bilinear map

$$
T\left(V^{\star}\right) \times T\left(V^{\star}\right) \rightarrow T\left(V^{\star}\right) .
$$


For example, the reader may check that

$$
(e \otimes f) ш g=e \otimes f \otimes g+e \otimes g \otimes f+g \otimes e \otimes f
$$

for any $e, f, g \in\left(\mathbf{R}^{2}\right)^{\star}$. For an alternative description of the shuffle product, see Definition 5.14 in [6].

Remark 4.4. It follows easily from the definition that the shuffle product is commutative and associative. We shall use the following notation for the shuffle product applied $N$ times:

$$
\mathbf{a}^{\amalg N}=\underbrace{\mathbf{a} \amalg \ldots \omega \mathbf{a}}_{N} .
$$

Later in $\S 5$ we shall also use the analogous version of the shuffle product defined on the tensor powers of $V$.

For each path $\gamma_{s}, s \in[0, T]$ of finite length, we now introduce a real-valued function

$$
\varphi(\gamma): T\left(V^{\star}\right) \rightarrow \mathbf{R}
$$

defined on the tensor $e=e^{i_{1}} \otimes \cdots \otimes e^{i_{n}}$ by

$$
\begin{aligned}
\varphi_{e}(\gamma) & =\left\langle e^{i_{1}} \otimes \cdots \otimes e^{i_{n}}, \int_{0<t_{1}<\cdots<t_{n}<T} d \gamma_{t_{1}} \otimes \cdots \otimes d \gamma_{t_{n}}\right\rangle \\
& =\int_{0<t_{1}<\cdots<t_{n}<T} d \gamma_{t_{1}}^{i_{1}} \ldots d \gamma_{t_{n}}^{i_{n}} .
\end{aligned}
$$

The fundamental property of $\varphi_{e}(\gamma)$ (see [12, Theorem 2.15]) is that

$$
\varphi_{e}(\gamma) \varphi_{f}(\gamma)=\varphi_{e ш f}(\gamma)
$$

As in the definition of signature, to extend the definition of $\varphi_{e}$ from smooth paths to Brownian paths, we replace the integral in (3) with a stochastic integral.

From now on we change slightly the notation for $e_{1}, e_{2}$ and $e^{1}, e^{2}$. Let $x, y$ be a basis for $\mathbf{R}^{2}$ and let $x^{*}, y^{*}$ be the dual basis of $\left(\mathbf{R}^{2}\right)^{\star}$. We use the techniques we have introduced to prove the following key theorem.

Theorem 4.5. The nth moment of Lévy area at time 1 for a two-dimensional Brownian motion $B$ starting at zero is the signature $\mathbf{X}_{0,1}(B)$ contracted with the shuffle powers of the dual tensor $\frac{1}{2}\left(x^{*} \otimes y^{*}-y^{*} \otimes x^{*}\right)$. That is,

$$
\mathbb{E}\left(\mathcal{A}^{n}\right)=2^{-2 n}\left\langle\left(x^{*} \otimes y^{*}-y^{*} \otimes x^{*}\right)^{\boldsymbol{w n}}, \frac{(x \otimes x+y \otimes y)^{\otimes n}}{n !}\right\rangle .
$$

Proof. Let $B=\left(X_{s}, Y_{s}\right), 0 \leq s \leq 1$ be a Brownian path in $\mathbf{R}^{2}$ starting at zero. Then

$$
\mathcal{A}=\frac{1}{2} \int_{0}^{1}\left(X_{s} d Y_{s}-Y_{s} d X_{s}\right)=\frac{1}{2} \int_{0<t<s<1}\left(d X_{t} d Y_{s}-d Y_{t} d X_{s}\right) .
$$

In a more canonical notation as in (3) we may write this as

$$
\mathcal{A}=\frac{1}{2} \int_{0<t_{1}<t_{2}<1}\left(d X_{t_{1}} d Y_{t_{2}}-d Y_{t_{1}} d X_{t_{2}}\right) .
$$


So $\mathcal{A}=\varphi_{\frac{1}{2}\left(x^{*} \otimes y^{*}-y^{*} \otimes x^{*}\right)}(B)=\frac{1}{2}\left(\varphi_{x^{*} \otimes y^{*}}(B)-\varphi_{y^{*} \otimes x^{*}}(B)\right)$. Further, using (44) we have

$$
\begin{aligned}
\mathcal{A}^{n} & =\frac{1}{2^{n}}\left\langle\left(x^{*} \otimes y^{*}-y^{*} \otimes x^{*}\right), \int_{0<t_{1}<t_{2}<1} d B_{t_{1}} \otimes d B_{t_{2}}\right\rangle^{n} \\
& =\frac{1}{2^{n}}\left\langle\left(x^{*} \otimes y^{*}-y^{*} \otimes x^{*}\right)^{\mathrm{wn}}, X_{0,1}^{2 n}(B)\right\rangle .
\end{aligned}
$$

Taking the expectation of $\mathcal{A}^{n}$ we have by (2),

$$
\begin{aligned}
\mathbb{E}\left(\mathcal{A}^{n}\right) & =2^{-n}\left\langle\left(x^{*} \otimes y^{*}-y^{*} \otimes x^{*}\right)^{\boldsymbol{w}}, \mathbb{E}\left(X_{0,1}^{2 n}(B)\right)\right\rangle \\
& =2^{-2 n}\left\langle\left(x^{*} \otimes y^{*}-y^{*} \otimes x^{*}\right)^{\boldsymbol{w} n}, \frac{(x \otimes x+y \otimes y)^{\otimes n}}{n !}\right\rangle .
\end{aligned}
$$

Hence to prove Theorem 2.1 it is sufficient to prove the following theorem.

\section{Theorem 4.6.}

$$
\left\langle\left(x^{*} \otimes y^{*}-y^{*} \otimes x^{*}\right)^{\boldsymbol{w}},(x \otimes x+y \otimes y)^{\otimes n}\right\rangle=2^{n} n ! E_{n} .
$$

\section{Proof of Theorem 4.6}

5.1. From now on it will often be convenient to use a shorter notation for elements in the standard basis of the tensor algebra $T(V)$, in which we write $x y$ rather than $x \otimes y, x^{2}$ rather than $x \otimes x$, and so on. Using this notation the standard basis elements of $T(V)$ are simply the words in the letters $x$ and $y$.

We shall say that a word in the letters $x$ and $y$ is even if (i) it is of the form $z_{1}^{2} \ldots z_{n}^{2}$ where each $z_{i} \in\{x, y\}$, and (ii) there are equal numbers of $x$ 's and $y$ 's. When we expand

$$
(x \otimes x+y \otimes y)^{\otimes n}
$$

we obtain the sum of all words in $x$ and $y$ of length $2 n$ satisfying condition (i). If such a word is not killed by $\left(x^{\star} \otimes y^{\star}-y^{\star} \otimes x^{\star}\right)^{\omega n}$, then clearly it must also satisfy (ii). Hence Theorem 4.6 is equivalent to the following assertion.

Theorem 5.1. Let

$$
(x y-y x)^{\boldsymbol{\omega} n}=\sum_{v} \lambda_{v} v
$$

where the sum is over all words $v$ of length $2 n$. Let $u_{n}=\sum \lambda_{w}$, where the sum is over all even words $w$ of length $2 n$. Then

$$
u_{n}=2^{n} n ! E_{n} .
$$

5.2. We begin the proof of Theorem 5.1 by noting that if $n$ is odd, then there are no even words of length $2 n$, and so $u_{n}=0$, as required. We may therefore assume that $n=2 m$ is even, so the even words that appear in $(x y-y x)^{\boldsymbol{m} n}$ are of length $4 m$, with the pairs $x x$ and $y y$ each appearing exactly $m$ times.

Our proof depends on counting the combinatorial objects introduced in the next definition.

Definition 5.2. An $x y$-matching is a pair $(w, \sigma)$, where $w$ is an even word, of length $4 m$ say, and $\sigma$ is a fixed-point-free involution in the symmetric group $S_{4 m}$ such that $w_{i}=x$ if and only if $w_{\sigma(i)}=y$. 


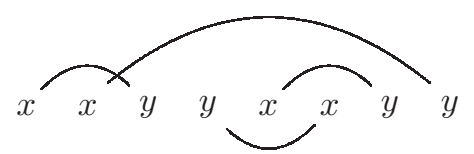

FiguRE 2. The $x y$-matching (xxyyxxyy,(13)(28)(46)(57)).

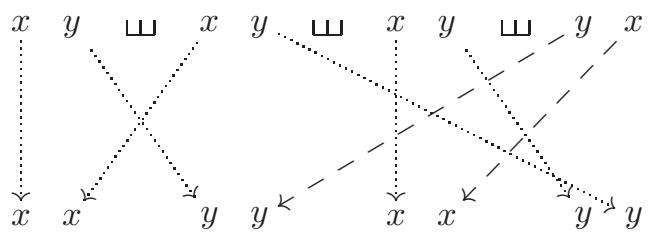

Figure 3. An expansion of $x y$ ш $x y \amalg x y \amalg y x$.

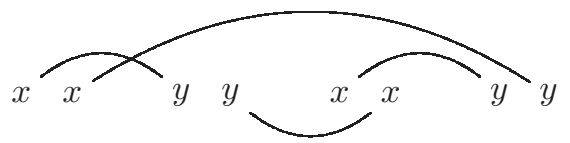

Figure 4 . The $x y$-matching corresponding to the expansion in Figure 3.

Given an $x y$-matching $\delta=(w, \sigma)$ we define the negativity of $\delta$ by

$$
\operatorname{neg}(\delta)=\#\left\{i \in\{1 \ldots 4 m\}: w_{i}=x \text { and } \sigma(i)<i\right\} .
$$

We define the sign of $\delta$ by $\operatorname{sgn}(\delta)=(-1)^{\operatorname{neg}(\delta)}$. Let $N_{t}(w)$ be the number of $x y$-matchings with underlying word $w$ and negativity $t$.

It will be very useful to represent $x y$-matchings by diagrams such as the one in Figure 2. The arcs contributing to neg $(\delta)$ are those drawn below the word, thus here $\operatorname{neg}(\delta)=1$ and $\operatorname{sgn}(\delta)=-1$. As an exercise, the reader may check that there are in total $16 x y$-matchings with underlying word xxyyxxyy and negativity 1 , and so $N_{1}($ xxyyxxyy) $=16$.

Proposition 5.3. Let $w$ be an even word of length $4 m$ and let $s+t=2 m$. The coefficient of $w$ in $(x y)^{山 s} \boldsymbol{\Psi}(y x)^{\boldsymbol{\omega} t}$ is $s ! t ! N_{t}(w)$.

The proof of this proposition is postponed to the appendix at the end of this paper. The idea is to associate to each $x y$-matching with underlying word $w$ and negativity $t$ exactly $s ! t$ ! ways to obtain $w$ by expanding the shuffle product $(x y)^{山 s} \amalg(y x)^{\omega t}$. With the help of a formal definition of an expansion of a shuffle product of this form, we are able to show that these possibilities are exhaustive. Here we shall illustrate the correspondence when $m=2, w=$ xxyyxxyy, $s=3$ and $t=1$.

Figure 3 shows one way to obtain $w$ by expanding the shuffle product $x y \amalg x y \amalg$ $x y \amalg y x$. We obtain the corresponding $x y$-matching (see Figure 4) by connecting the letters coming from the same $x y$ or $y x$ term in $x y \omega x y 山 x y 山 y x$. Note that this matching, (xxyyxxyy,(13)(28)(46)(57)), has negativity 1, corresponding to the single $y x$ term.

There are in total 3 !1! ways to obtain $w$ by expanding the shuffle product $x y \amalg x y \amalg x y \amalg y x$ which correspond to this matching. The remaining five are 


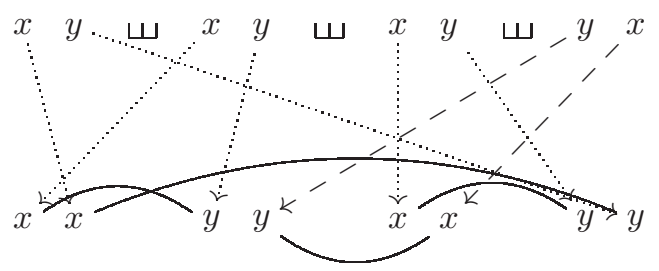

FiguRE 5. Another of the 3!1! expansions of $x y \amalg x y \amalg x y \amalg y x$ giving the $x y$-matching shown in Figure 4.

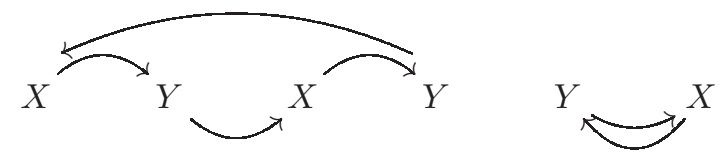

Figure 6 . The $X Y$-matching $(X Y X Y Y X,(1234)(56))$.

obtained by permuting identical words in the top line of Figure 3. For example, we get the same matching by expanding the shuffle product as shown in Figure 5 .

Corollary 5.4. Let $w$ be an even word of length $4 m$. The coefficient of $w$ in $(x y-y x)^{\mathbf{\omega} 2 m}$ is

$$
(2 m) ! \sum_{\delta} \operatorname{sgn}(\delta)
$$

where the sum is over all xy-matchings $\delta$ with underlying word $w$. Hence

$$
u_{2 m}=(2 m) ! \sum_{\delta} \operatorname{sgn}(\delta),
$$

where the sum is over all xy-matchings $\delta$ with underlying word of length $4 m$.

Proof. Let $s+t=2 m$. As the shuffle product is commutative, when we expand $(x y-y x)^{山 2 m}$ we obtain $(-1)^{t}(x y)^{山 s} \boldsymbol{\omega}(y x)^{\boldsymbol{\omega} t}$ exactly $\left(\begin{array}{c}2 m \\ s\end{array}\right)$ times. Hence, by Proposition 5.3, the coefficient we seek is

$$
\sum_{s+t=2 m}\left(\begin{array}{c}
2 m \\
s
\end{array}\right)(-1)^{t} s ! t ! N_{t}(w)=(2 m) ! \sum_{t=0}^{2 m}(-1)^{t} N_{t}(w)=(2 m) ! \sum_{\delta} \operatorname{sgn}(\delta),
$$

where the final equality holds because the middle sum counts each $x y$-matching exactly once, with the appropriate sign. The equation for $u_{2 m}$ now follows by summing over all even words of length $4 m$.

5.3. We now introduce our second and final combinatorial object.

Definition 5.5. An $X Y$-matching is a pair $(W, \sigma)$ where $W$ is a word in letters $X$ and $Y$ with equal numbers of $X$ 's and $Y$ 's, say $m$ of each, and $\sigma \in S_{2 m}$ is a permutation such that $W_{i}=X$ if and only if $W_{\sigma(i)}=Y$.

Once again, it is very useful to represent $X Y$-matchings by diagrams. Figure 6 shows a typical example. As before, we need certain quantities associated with an $X Y$-matching.

Definition 5.6. Let $\Delta=(W, \sigma)$ be an $X Y$-matching. The length of $\Delta$ is the length of the word $W$. We define the negativity of $\Delta$ by

$$
\operatorname{neg}(\Delta)=\#\left\{i: W_{i}=X \text { and } \sigma(i)<i \text { or } W_{i}=Y \text { and } \sigma(i)>i\right\} \text {. }
$$



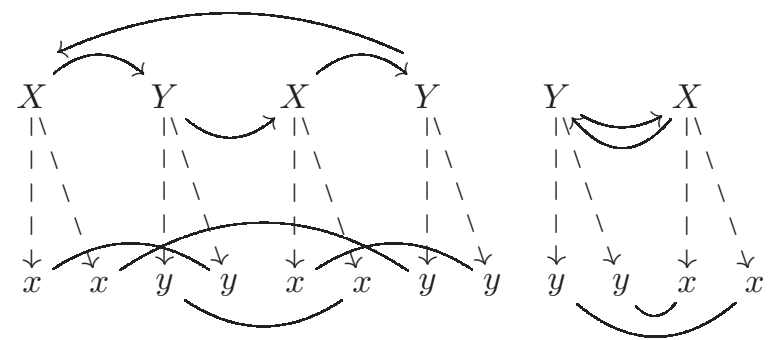

Figure 7 . The canonical $x y$-matching associated to $(W, \sigma)$.

We define the sign of $\Delta$ by

$$
\operatorname{sgn}(\Delta)=(-1)^{\operatorname{neg}(\Delta)} .
$$

We define the cycle count of $\Delta$ by

$$
\operatorname{cyc}(\Delta)=\# \text { disjoint cycles in the permutation } \sigma .
$$

Finally we let $e(\Delta)$ be the even word obtained from $\Delta$ by replacing each $X$ with $x x$ and each $Y$ with $y y$.

For example, if $\Delta$ is the $X Y$-matching shown in Figure 6, then $\Delta$ has length 6 , $\operatorname{neg}(\Delta)=3$ (the 3 arcs drawn below the word contributing), $\operatorname{sgn}(\Delta)=-1, \operatorname{cyc}(\Delta)=$ 2 and $e(\Delta)=$ xxy $x$ xxyyy $x x$.

Proposition 5.7. Let $w$ be an even word of length $4 m$ and let $s+t=2 m$. The coefficient of $w$ in $(x y)^{\boldsymbol{\omega} s} \boldsymbol{\omega}(y x)^{\mathrm{\omega t}}$ is

$$
2^{2 m} s ! t ! \sum_{\Delta} 2^{-\operatorname{cyc}(\Delta)},
$$

where the sum is over all the $X Y$-matchings $\Delta$ such that $e(\Delta)=w$ and $\operatorname{neg}(\Delta)=t$.

Proof. By Proposition 5.3. it is sufficient to prove that

$$
N_{t}(w)=2^{2 m} \sum_{\Delta} 2^{-\operatorname{cyc}(\Delta)},
$$

where the sum is over all the $X Y$-matchings $\Delta$ such that $e(\Delta)=w$ and neg $(\Delta)=t$. To do this, we shall associate to each $X Y$-matching $\Delta=(W, \sigma)$ exactly $2^{2 m-\operatorname{cyc}(\Delta)}$ $x y$-matchings with underlying word $e(\Delta)$ and negativity equal to neg $(\Delta)$.

The canonical such $x y$-matching is $\delta=(e(W), \tau)$, where $\tau$ is the involution defined by

$$
\tau(2 i-1)=2 \sigma(i) \quad \text { if } 1 \leq i \leq 2 m .
$$

For example, if $W=X Y X Y Y X$ and $\sigma=(1234)(56)$, then

$$
\tau=(14)(27)(36)(58)(912)(1011) .
$$

as shown in Figure 7.

We obtain the remaining $x y$-matchings by conjugating $\tau$ by the $2^{2 m}$ elements of the group $\langle(12), \ldots,(4 m-14 m)\rangle$. There is, however, some double counting, which accounts for the factor of $2^{-\operatorname{cyc}(\Delta)}$. There are two cases we must consider.

Firstly, suppose $\sigma$ has a 2-cycle, say $(k l)$. Then $\tau$ involves

$$
(2 k-1 \quad 2 l)(2 k \quad 2 l-1) \text {, }
$$


which is stabilised by conjugation by $(2 k-12 k)(2 l-12 l)$. This gives us a factor of $1 / 2$ for each 2-cycle.

Secondly, if $\hat{\sigma}$ is a cycle of length 4 or more in $\sigma$, then we can replace $\hat{\sigma}$ with $\hat{\sigma}^{-1}$ without changing the set of $x y$-matchings we obtain. So again we must compensate by a factor of $1 / 2$ to avoid overcounting. For example, the two $X Y$-matchings $(X Y X Y,(1234))$ and $(X Y X Y,(1432))$ both give the same set of $16 x y$-matchings. Combining these observations gives the required result.

By the same argument used to deduce Corollary 5.4 from Proposition 5.3 we obtain the following corollary.

Corollary 5.8. Let $w$ be an even word of length $4 m$. The coefficient of $w$ in $(x y-y x)^{\mathrm{\omega} 2 m}$ is

$$
(2 m) ! 2^{2 m} \sum_{\Delta} 2^{-\operatorname{cyc}(\Delta)} \operatorname{sgn}(\Delta),
$$

where the sum is over all $X Y$-matchings $\Delta$ such that $e(\Delta)=w$. Hence

$$
u_{2 m}=(2 m) ! 2^{2 m} \sum_{\Delta} 2^{-\operatorname{cyc}(\Delta)} \operatorname{sgn}(\Delta),
$$

where the sum is over all $X Y$-matchings $\Delta$ of length $2 m$.

5.4. Let

$$
c_{2 r}=\sum_{\Delta} \operatorname{sgn}(\Delta)
$$

where the sum is over all $X Y$-matchings $\Delta$ of length $2 r$ with just one cycle. We use an argument from the theory of exponential structures (see [13, §5.4] for the general setting) to obtain an expression for $u_{2 m}$ in terms of the $c_{2 r}$. This reduces our problem to finding the $c_{2 r}$.

\section{Lemma 5.9.}

$$
u_{2 m}=(2 m) ! 2^{2 m} \sum_{\substack{a_{1}, \ldots, a_{m} \geq 0 \\ a_{1}+2 a_{2}+\ldots+m a_{m}=m}} \frac{(2 m) !}{(2 !)^{a_{1}} \ldots(2 m) !^{a_{m}}} \frac{c_{2}^{a_{1}}}{2^{a_{1}} a_{1} !} \cdots \frac{c_{2 m}^{a_{m}}}{2^{a_{m}} a_{m} !} .
$$

Proof. Consider the contribution to the sum in (5) coming from those $X Y$-matchings whose underlying permutation has cycle type $\left(2^{a_{1}}, \ldots,(2 m)^{a_{m}}\right)$. (Here the exponent $a_{i}$ gives the number of cycles of length $2 i$.) To construct such an $X Y$-matching, we must first partition $\{1 \ldots 2 m\}$ into $a_{1}$ subsets of size $2, a_{2}$ subsets of size 4 , and so on, up to $a_{m}$ subsets of size $2 m$. This can be done in

$$
\frac{(2 m) !}{(2 !)^{a_{1}} a_{1} ! \ldots(2 m) !^{a_{m}} a_{m} !}
$$

ways. (The factors $a_{1} !, \ldots, a_{m} !$ appear because we do not care in which order we choose the $a_{r}$ subsets of size $r$.)

Then we must choose for each subset of size $2 r$ an $X Y$-matching with just one cycle on that subset. The sign of the resulting $X Y$-matching is the product of the signs of the $X Y$-matchings on the subset, and the total number of cycles is $a_{1}+\cdots+a_{m}$. By Corollary 5.8, the contribution to $u_{2 m}$ coming from this particular choice of subsets is

$$
(2 m) ! 2^{2 m} \frac{c_{2}^{a_{1}} \ldots c_{2 m}^{a_{m}}}{2^{a_{1}} \ldots 2^{a_{m}}} .
$$


Hence the total contribution to $u_{2 m}$ coming from permutations with cycle type $\left(2^{a_{1}}, \ldots,(2 m)^{a_{m}}\right)$ is

$$
(2 m) ! 2^{2 m} \frac{(2 m) !}{(2 !)^{a_{1}} \ldots(2 m) !^{a_{m}}} \frac{c_{2}^{a_{1}}}{2^{a_{1}} a_{1} !} \cdots \frac{c_{2 m}^{a_{m}}}{2^{a_{m}} a_{m} !} .
$$

The result now follows by summing over all possible cycle types.

Lemma 5.10. Let

$$
f(z)=\sum_{m=0}^{\infty} \frac{u_{2 m}}{2^{2 m}(2 m) !} \frac{z^{2 m}}{(2 m) !}
$$

be the exponential generating function of $\frac{u_{2 m}}{2^{2 m}(2 m) !}$. Then

$$
f(z)=\exp \left(\sum_{r=1}^{\infty} \frac{c_{2 r}}{2} \frac{z^{2 r}}{(2 r) !}\right) .
$$

Proof. This is an immediate consequence of Lemma 5.9 .

5.5. To finish the proof we need to know the $c_{2 r}$. Since the sign of an $X Y$-matching is not affected by swapping the letters $X$ and $Y$, we have

$$
c_{2 r}=2 \sum_{\Delta} \operatorname{sgn}(\Delta)
$$

where the sum is over all $X Y$-matchings $\Delta$ of length $2 r$ such that $\operatorname{cyc}(\Delta)=1$ and the first letter of $W$ is $X$.

To evaluate this sum we need the following lemma. Recall that a permutation $\tau$ is said to have a descent at $i$ if $\tau(i)>\tau(i+1)$.

Lemma 5.11. Let $\Delta=(W, \sigma)$ be an $X Y$-matching of length $2 r$ such that $\operatorname{cyc}(\Delta)=$ 1 and $W_{1}=X$. Suppose that $\sigma=\left(\begin{array}{lll}1 & b_{1} \ldots b_{2 r-1}\end{array}\right)$. Let $\tau \in S_{\{2 \ldots 2 m\}}$ be the permutation defined by $\tau(i)=b_{i}$. Then

$$
\operatorname{sgn}(\Delta)=(-1)^{r-1}(-1)^{\operatorname{desc}(\tau)}
$$

where $\operatorname{desc}(\tau)$ is the number of descents in $\tau$.

Proof. If $i$ is even, then $W_{b_{i}}=X$, and if $i$ is odd, then $W_{b_{i}}=Y$. Hence

$$
\operatorname{neg}(\Delta)=\#\left\{i: i \text { is even and } b_{i+1}<b_{i}\right\}+\#\left\{i: i \text { is odd and } b_{i+1}>b_{i}\right\} \text {. }
$$

But \#\{i:i is odd and $\left.b_{i+1}>b_{i}\right\}+\#\left\{i: i\right.$ is odd and $\left.b_{i+1}<b_{i}\right\}=r-1$ as there are $r-1$ odd numbers $i$ such that $1 \leq i<2 r-1$. Hence

$$
\begin{array}{r}
\#\left\{i: i \text { is odd and } b_{i+1}>b_{i}\right\} \equiv \#\left\{i: i \text { is odd and } b_{i+1}<b_{i}\right\} \\
+(r-1) \bmod 2 .
\end{array}
$$

The result now follows.

Let $\left\langle\begin{array}{l}t \\ d\end{array}\right\rangle$ denote the number of permutations in $S_{t}$ with exactly $d$ descents. (These are known as the Eulerian numbers; our notation for them is taken from [7, §5.1.3].) By the previous lemma,

$$
c_{2 r}=2(-1)^{r-1} \sum_{d=0}^{2 r-1}(-1)^{d}\left\langle\begin{array}{c}
2 r-1 \\
d
\end{array}\right\rangle
$$


for each $r \in \mathbf{N}$. By a well-known property of Eulerian numbers (see, for instance, [7. Exercise 5.1.3(3)]) we have

$$
\sum_{d=0}^{2 r-1}(-1)^{d}\left\langle\begin{array}{c}
2 r-1 \\
d
\end{array}\right\rangle=(-1)^{r-1} T_{r},
$$

where the $T_{r}$ are the tangent numbers, defined by

$$
\sum_{r=1}^{\infty} T_{r} \frac{z^{2 r-1}}{(2 r-1) !}=\tan z
$$

We have therefore shown that

$$
c_{2 r}=2 T_{r} \quad \text { for all } r \in \mathbf{N} .
$$

5.6. By (6) and (77),

$$
\begin{array}{r}
f(z)=\exp \left(\sum_{r=1}^{\infty} T_{r} \frac{z^{2 r}}{(2 r) !}\right)=\exp \left(\int \sum_{r=1}^{\infty} T_{r} \frac{z^{2 r-1}}{(2 r-1) !}\right) \\
=\exp \left(\int \tan z\right)=\exp (\log \sec z)=\sec z .
\end{array}
$$

The right hand side is the exponential generating function for the Euler numbers, so comparing coefficients, we see that $\frac{u_{2 m}}{2^{2 m}(2 m) !}=E_{2 m}$. This completes the proof.

\section{Appendix: Proof of Proposition 5.3}

We repeat the statement of this proposition below.

Proposition 5.3. Let $w$ be an even word of length $4 m$ and let $s+t=2 m$. The coefficient of $w$ in $(x y)^{\boldsymbol{\omega} s} \boldsymbol{\amalg}(y x)^{\boldsymbol{\omega} t}$ is $s ! t ! N_{t}(w)$.

We urge the reader to read the discussion following the original statement of this proposition in $\S 5.2$ before proceeding. What follows is a formalised version of the argument we indicated there.

Proof. By an expansion of $(x y)^{山 s} \amalg(y x)^{\omega t}$ we mean a word of length $4 m$ using each of the letters

$$
x^{1}, y^{1}, \ldots, x^{s}, y^{s}, y_{1}, x_{1}, \ldots y_{t}, x_{t}
$$

exactly once, and such that for each $i, x^{i}$ appears before $y^{i}$, and $x_{i}$ appears after $y_{i}$. It should be clear that the coefficient of $w$ in $(x y)^{\omega s} \omega(y x)^{\omega t}$ is equal to the number of expansions of $(x y)^{\boldsymbol{山} s} \amalg(y x)^{\boldsymbol{}}{ }^{t}$ which become $w$ when the numbers attached to the letters are erased.

The orbits of $S_{s} \times S_{t}$ on expansions of $(x y)^{\boldsymbol{\omega} s} \amalg(y x)^{山 t}$ are all of size s!t!. Given such an orbit we shall define a corresponding $x y$-matching with underlying word $w$, and permutation $\sigma$. Choose any representative of the orbit, $w^{\star}$ say. For $k \in\{1 \ldots 2 m\}$ set $\sigma(k)=l$, where $l$ is defined by

$$
\text { if }\left\{\begin{array}{l}
w_{k}^{\star}=x_{i}, \text { then } w_{l}^{\star}=y_{i}, \\
w_{k}^{\star}=x^{i}, \text { then } w_{l}^{\star}=y^{i}, \\
w_{k}^{\star}=y_{i}, \text { then } w_{l}^{\star}=x_{i}, \\
w_{k}^{\star}=y^{i}, \text { then } w_{l}^{\star}=x^{i} .
\end{array}\right.
$$


It is easy to check that $\sigma$ is a fixed-point-free involution, that $\sigma$ does not depend on the choice of $w^{\star}$, and that $\operatorname{neg}(w, \sigma)=t$.

Conversely, suppose we are given an $x y$-matching with underlying word $w$ and permutation $\sigma$ and negativity $t$. Set $s=2 m-t$. There is a unique way to write $\sigma$ in the form

$$
\left(i^{1} \sigma\left(i^{1}\right)\right) \ldots\left(i^{s} \sigma\left(i^{s}\right)\right)\left(\sigma\left(i_{1}\right) i_{1}\right) \ldots\left(\sigma\left(i_{t}\right) i_{t}\right)
$$

such that the following conditions hold:

$$
\begin{aligned}
& \text { (1) } i^{1}<i^{2}<\ldots<i^{s}, \quad w_{i^{j}}=x, \quad \sigma\left(i^{j}\right)>i^{j} \quad \text { if } 1 \leq j \leq s \\
& \text { (2) } i_{1}<i_{2}<\ldots<i_{t}, \quad w_{i_{k}}=x, \quad \sigma\left(i_{k}\right)<i_{k} \text { if } 1 \leq k \leq t .
\end{aligned}
$$

We shall define an associated expansion $w^{\star}$ of $(x y)^{山 s} \amalg(y x)^{\boldsymbol{\omega} t}$. The underlying word of $w^{\star}$ is, of course, $w$. For $j \in\{1, \ldots, s\}$ we set $w_{i^{j}}^{\star}=x^{j}$ and $w_{\sigma\left(i^{j}\right)}^{\star}=y^{j}$. For $k \in\{1, \ldots, t\}$ we set $w_{i_{j}}^{\star}=x_{j}$ and $w_{\sigma\left(i_{j}\right)}^{\star}=y_{j}$. By virtue of our expression for $\sigma$, the expansion $w^{\star}$ we have defined is canonical, in the sense that the subscripts and superscripts on its letters appear in increasing order.

Clearly each $S_{s} \times S_{t}$-orbit contains a unique canonical expansion. Hence to prove the proposition it is sufficient to prove that the two maps we have defined give a bijection between $x y$-matchings and canonical expansions. This is merely a matter of definition chasing. We illustrate it by an example.

Suppose $\delta=($ xxyyxxyy,$(13)(28)(46)(57))$. As $\operatorname{neg}(\delta)=1$, we associate to $\delta$ an expansion of $x y 山 x y 山 x y 山 y x$. Following the given algorithm, we take $i^{1}=$ $1, i^{2}=2, i^{3}=5$ and $i_{1}=6$ and assign the labels $\left(x^{1} x^{2} y^{1} y_{1} x^{3} x_{1} y^{3} y^{2}\right)$. Conversely, given this expansion, we get back the permutation (13)(28)(46)(57).

\section{ACKNOWLEDGEMENTS}

The first-named author wishes to thank Thierry Lévy and Terry Lyons for valuable discussions. The authors would also like to thank the anonymous referee for his or her helpful comments on the first version of this paper.

\section{REFERENCES}

[1] Chen, K.-T. Integration of paths, geometric invariants and a generalized Baker-Hausdorff formula, Ann. of Math. (2) 65 (1957), 163-178. MR0085251(19:12a)

[2] O'Connell, N. Conditioned random walks and the RSK correspondence. Random matrix theory, J. Phys. A 36 (2003), no. 12, 3049-3066. MR1986407 (2004e:05201)

[3] FAwCETt, T. Problems in stochastic analysis. Connections between rough paths and noncommutative harmonic analysis. D. Phil. thesis, Oxford University, 2003.

[4] Gaveau, B. Principe de moindre action, propagation de la chaleur et estimées sous elliptiques sur certains groupes nilpotents, Acta Math. 139 (1977), no. 1-2, 95-153. MR0461589 $(57: 1574)$

[5] Gaines, J. G. The algebra of iterated stochastic integrals, (English summary) Stochastics Stochastics Rep. 49 (1994), no. 3-4, 169-179. MR1785003 (2001e:60113)

[6] Hambly, B. And Lyons, T. Uniqueness for the signature of a path of bounded variation and continuous analogues of the free group, arXiv:math.CA/0507536 (submitted).

[7] Knuth, D. E. The art of computer programming. Volume 3. Addison-Wesley Publishing Co., Reading, Mass.-London-Don Mills, Ont., 1973. Sorting and searching, Addison-Wesley Series in Computer Science and Information Processing. MR0445948 (56:4281)

[8] LÉvy, P. Le mouvement Brownien plan. (French) Amer. J. Math. 62 (1940), 487-550. MR0002734 (2:107g)

[9] Lévy, P. Processus stochastiques et mouvement Brownien, Suivi d'une note de M. Loève. (French) Gauthier-Villars, Paris, 1948. MR0190953 (32:8363) 
[10] LÉVy, P. Wiener's random function, and other Laplacian random functions, in Proceedings of the Second Berkeley Symposium on Mathematical Statistics and Probability, 1950, 171187, Univ. California Press, Berkeley and Los Angeles, 1951. MR 0044774 (13:476b)

[11] Lyons, T. And Victoir, N. Cubature on Wiener space, Proc. R. Soc. Lond. Ser. A Math. Phys. Eng. Sci. 460 (2004), no. 2041, 169-198. MR2052260(2005b:35306)

[12] Lyons, T. J., LÉvy, T., And Caruana, M. Differential equations driven by rough paths, vol. 1908 of Lecture Notes in Mathematics Ecole d'Eté de Probabilités de Saint-Flour XXXIV2004, Springer, 2007. MR2314753

[13] Stanley, R. P. Enumerative combinatorics. Vol. 2, vol. 62 of Cambridge Studies in Advanced Mathematics. Cambridge University Press, Cambridge, 1999. With a foreword by Gian-Carlo Rota and an appendix by Sergey Fomin. MR1676282 (2000k:05026)

Mathematical Institute, University of Oxford, 24-29 St Giles', Oxford OX1 3LB, UNITED KingDOM

E-mail address: levin@maths.ox.ac.uk

Department of Mathematics, University of Wales, Swansea, Singleton Park, Swansea SA2 8PP, United Kingdom

E-mail address: m.j.wildon@swansea.ac.uk 